\title{
Knots in lens spaces with the 3-sphere surgery
}

\author{
TOSHIO SAITO
}

\begin{abstract}
In this paper, we improve a necessary condition which we gave in [17], for 1-bridge braids in lens spaces to admit an integral surgery yielding the 3-sphere. As an application, we prove that if the lens space of type $(p, q)$ is obtained by Berge's surgery on a nontrivial nontorus doubly primitive knot in the 3-sphere, then $|q| \geq 5$. To this end, we completely list up all such lens spaces with $|q|<5$ and prove that they are obtained only by torus knots.
\end{abstract}

57M27; 57M25

\section{Introduction}

Let $N$ be a compact 3-manifold and $K$ a knot in $N$. Let $m$ be a meridian of $K$ in $\partial \eta(K ; N)$, where $\eta(B ; A)$ is a regular neighborhood of $B$ in $A$. We fix a longitude $\ell$ in $\partial \eta(K ; N)$ such that $m$ intersects $\ell$ transversely in a single point. When $N$ is homeomorphic to the 3 -sphere $S^{3}, \ell$ should be the preferred longitude of $K$ in $\partial \eta(K ; N)$. In the following, we fix an orientation of $m$ and $\ell$ as illustrated in Figure 1. A Dehn surgery on $K$ is an operation where we attach a solid torus $\bar{V}$ to $E(K ; N)$ by a boundary-homeomorphism $\varphi: \partial \bar{V} \rightarrow \partial E(K ; N)$, where $E(B ; A)$ means the exterior of $B$ in $A$, ie, $E(B ; A)=\operatorname{cl}(A \backslash \eta(B ; A))$. If $\varphi(\bar{m})$ is isotopic to a simple loop represented by $p[m]+q[\ell]$ for a meridian $\bar{m}$ of $\bar{V}$, then the surgery is called $p / q$-surgery. By an integral surgery, we mean a Dehn surgery such that $p / q$ is an integer. The 3 -manifold obtained by $p / q$-surgery on a trivial knot in $S^{3}$ is called the lens space of type $(p, q)$ and is denoted by $L(p, q)$. Note that $L(-p,-q) \cong L(p, q) \cong L(p, q+n p)$ for any integer $n$. Hence we may assume that $p>0$ and $0 \leq q<p$ except for $L(0,1)=S^{2} \times S^{1}$. In this paper, a lens space surgery means a Dehn surgery yielding a lens space, and the 3 -sphere surgery, $S^{3}$-surgery briefly, means a Dehn surgery yielding $S^{3}$.

It is an interesting problem on Dehn surgeries to decide the types of lens spaces which are obtained by a Dehn surgery on a nontrivial knot in $S^{3}$. We remark that the problem was completely solved for torus knots by Moser [14] and satellite knots by Bleiler and Litherland [2], Wang [18] and Wu [19]. Also, there are hyperbolic knots with lens space surgeries. Such examples were first found by Fintushel and Stern [6]. They proved that 
18 - and 19-surgery on the $(-2,3,7)$-pretzel knot yield the lens spaces $L(18,5)$ and $L(19,7)$ respectively. By the Cyclic Surgery Theorem of Culler, Gordon, Luecke and Shalen [5], a lens space surgery on a nontrivial nontorus knot must be integral. Gordon and Luecke showed that nontrivial surgery on a nontrivial knot cannot yield $S^{3}$ [8], and Gabai proved that $S^{2} \times S^{1}$ never comes from Dehn surgeries on a nontrivial knot [7]. In [1], Berge introduced the concept of doubly primitive knots and showed that there is an integral surgery on a doubly primitive knot yielding a lens space. In this paper, we call such a surgery Berge's surgery (for details, see the appendix of [17]).

Definition 1.1 (Berge) Let $\left(V_{1}, V_{2} ; S\right)$ be a genus two Heegaard splitting of $S^{3}$ and $K$ a simple loop on $S$. Then $K$ is called a doubly primitive knot if $K$ represents a free generator both of $\pi_{1}\left(V_{1}\right)$ and of $\pi_{1}\left(V_{2}\right)$.

Precisely, Berge's surgery is a Dehn surgery along a surface slope when a knot is put in a doubly primitive position. Hence Berge's surgery is always integral. We remark that it remains possible that a doubly primitive knot admits a lens space surgery other than Berge's surgery. However, it is conjectured by Gordon [12, Problem 1.78] that if a lens space is obtained by a Dehn surgery on a nontrivial nontorus knot, then it would be obtained only by Berge's surgery on a doubly primitive knot. The following conjecture is given by Bleiler and Litherland [2].

Conjecture 1.2 It would be impossible to obtain a lens space $L(p, q)$ with $|p|<18$ by a Dehn surgery on a nontrivial nontorus knot.

In [17], we showed that the conjecture is true for nontrivial nontorus doubly primitive knots in $S^{3}$ if $L(p, q)$ is obtained by Berge's surgery.

Based on this background, we consider the following question given by Ichihara in private communication.

Question 1.3 Is it impossible to obtain a lens space $L(p, q)$ with $|q|<5$ by a Dehn surgery on a nontrivial nontorus knot?

Note that $L(1,0)=S^{3}$ and hence the question is true for $q=0$ by Gordon and Luecke [8]. Hirasawa and Shimokawa [9] showed that a Dehn surgery on strongly invertible knots cannot give $L(2 p, 1)$ for any integer $p$. In [13], Kronheimer, Mrowka, Ozsváth and Szabó proved that $L(p, 1)$ cannot be obtained by $p$-surgery on a nontrivial knot. In [15], Rasmussen showed that $L(p, 2)$ can be obtained by $p$-surgery on a knot only if $p=7$ and that $L(p, 3)$ can be obtained by $p$-surgery on a knot only if $p=11$ and 13. In each case, moreover, it is realized only by a torus knot. On the other hand, as previously mentioned, $L(18,5)$ is obtained by 18 -surgery on the $(-2,3,7)$-pretzel knot. In this paper, we prove the following which is a partial answer to Question 1.3. 
Theorem 1.4 Let $K$ be a nontrivial nontorus doubly primitive knot in $S^{3}$ and $L(p, q)$ a lens space obtained by Berge's surgery on $K$. Then $|q| \geq 5$.

In fact, we completely list up all such lens spaces with $|q|<5$ and prove that they are obtained only by torus knots. See Section 2 for the precise statement.

\section{Statement of results}

Let $K$ be a knot in a 3-manifold $N$ and $N^{\prime}$ a 3-manifold obtained by a Dehn surgery on $K$. Then $N^{\prime}=E(K ; N) \cup \bar{V}$, where $\bar{V}$ is an attaching solid torus. Let $K^{*} \subset N^{\prime}$ be a core loop of $\bar{V}$. We call $K^{*}$ the dual knot of $K$ in $N^{\prime}$. We remark that $E(K ; N)$ is homeomorphic to $E\left(K^{*} ; N^{\prime}\right)$ and that if integral surgery on $K$ in $N$ yields a 3manifold $N^{\prime}$, then $K^{*} \subset N^{\prime}$ admits integral surgery yielding $N$. We remark that a core knot in a lens space is the dual knot of a trivial knot in $S^{3}$, where a core knot is a knot whose exterior is homeomorphic to a solid torus.

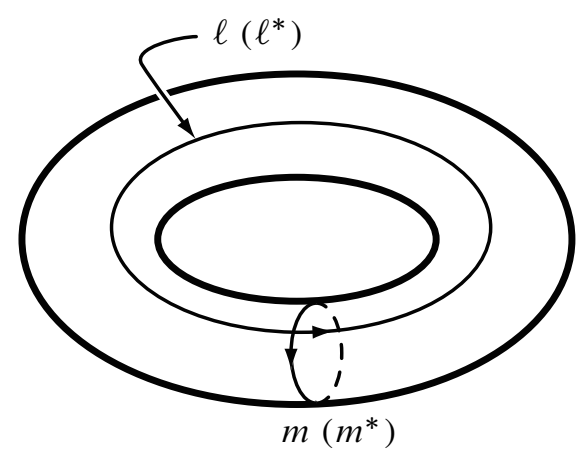

Figure 1: Once a knot is oriented, a longitude $\ell$ (resp. $\left.\ell^{*}\right)$ is oriented in the same direction as the knot. Then a meridian $m$ (resp. $m^{*}$ ) is oriented as in this figure.

Definition 2.1 Let $V_{1}$ be a regular neighborhood of a trivial knot in $S^{3}, m$ a meridian of $V_{1}$ and $\ell$ a longitude of $V_{1}$ such that $\ell$ bounds a disk in $\operatorname{cl}\left(S^{3} \backslash V_{1}\right)$. We fix an orientation of $m$ and $\ell$ as illustrated in Figure 1. By attaching a solid torus $V_{2}$ to $V_{1}$ so that $\bar{m}$ is isotopic to a representative of $p[\ell]+q[m]$, we obtain a lens space $L(p, q)$, where $p$ and $q$ are coprime integers and $\bar{m}$ is a meridian of $V_{2}$. The intersection points of $m$ and $\bar{m}$ are labelled $P_{0}, \ldots, P_{p-1}$ successively along the positive direction of $m$. Let $t_{i}^{u}(i=1,2)$ be simple arcs in $D_{i}$ joining $P_{0}$ to $P_{u}(u=1,2, \ldots, p-1)$. Then the notation $K(L(p, q) ; u)$ denotes the knot $t_{1}^{u} \cup t_{2}^{u}$ in $L(p, q)$ (see Figure 2). 
It is proved in [1] that when Berge's surgery on a doubly primitive knot yields a lens space, its dual knot is isotopic to a knot defined as $K^{*}=K(L(p, q) ; u)$ (see also Section 6 of [17]).

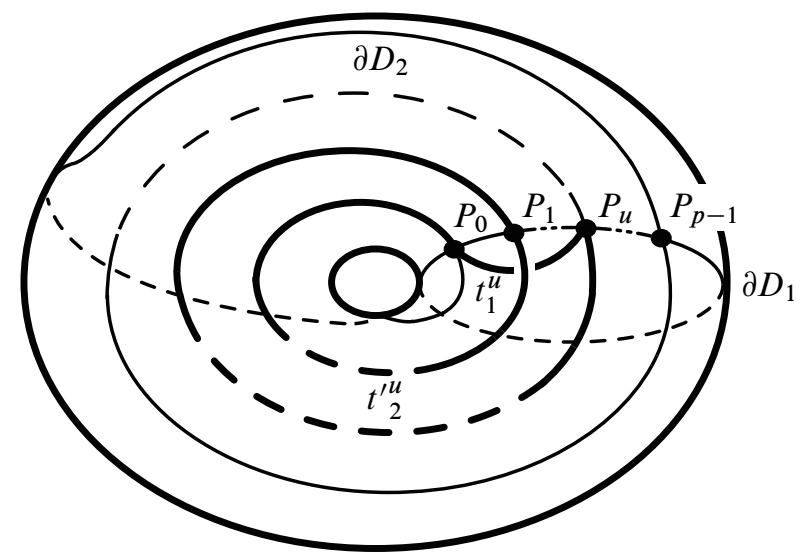

Figure 2: Here, $t_{2}^{\prime \prime}$ is a projection of $t_{2}^{u}$ on $\partial V_{1}$.

Remark 2.2 We note that two lens spaces $L(p, q)$ and $L\left(p^{\prime}, q^{\prime}\right)$ are (possibly orientation reversing) homeomorphic if and only if $|p|=\left|p^{\prime}\right|$, and $q \equiv \pm q^{\prime}(\bmod p)$ or $q q^{\prime} \equiv$ $\pm 1(\bmod p)$. Also, we easily see that $K(L(p, q) ; u)$ is isotopic to $K(L(p, q) ; p-u)$. Hence for $K(L(p, q) ; u)$, we often assume that $0<q<p / 2$ and $1 \leq u \leq p / 2$ in the remainder of the paper. We further remark that it is not always true that a knot represented by $K(L(p, q) ; u)$ admits integral $S^{3}$-surgery.

Throughout this section, we use the notation in Definition 2.1. Recall that we assume $p>1$ and $0<q<p$. Let $D_{1}\left(D_{2}\right.$ resp.) be a meridian disk in $V_{1}\left(V_{2}\right.$ resp.) with $\partial D_{1}=m$ and $\left|\partial D_{1} \cap \partial D_{2}\right|=p$. Let $t_{1}^{\prime u}\left(t_{2}^{\prime \prime}\right.$ resp.) be the arc in $\partial D_{1}\left(\partial D_{2}\right.$ resp.) whose initial point is $P_{0}$ and whose endpoint is $P_{u}$ passing in the positive direction of $m$ ( $\ell$ resp.). Then ${t^{\prime}}_{1}^{\prime u}\left(t_{2}^{\prime \prime}{ }_{2}\right.$ resp.) is a projection of $t_{1}^{u}\left(t_{2}^{u}\right.$ resp.). Set $V_{1}^{\prime}=V_{1} \cup \eta\left(t_{2}^{u} ; V_{2}\right), V_{2}^{\prime}=\operatorname{cl}\left(V_{2} \backslash \eta\left(t_{2}^{u} ; V_{2}\right)\right)$ and $S^{\prime}=\partial V_{1}^{\prime}=\partial V_{2}^{\prime}$. Then $V_{1}^{\prime}$ and $V_{2}^{\prime}$ are genus two handlebodies. Let $D_{2}^{\prime} \subset\left(D_{2} \cap V_{2}^{\prime}\right)$ be a meridian disk of $V_{2}^{\prime}$ with $\partial D_{2}^{\prime} \supset\left(t_{2}^{\prime}{ }_{2}^{u} \cap S^{\prime}\right)$. Let $m^{\prime}$ be a core loop of the annulus $\partial \eta\left(t_{2}^{u} ; V_{2}\right)$. Let $\ell^{\prime}$ be an essential loop in $S^{\prime}$ which is a union of ${t^{\prime}}_{1}^{\prime \prime} \cap S^{\prime}$ and an essential arc in the annulus $S^{\prime} \cap \partial \eta\left(t_{2}^{u} ; V_{2}\right)$ disjoint from $\partial D_{2}^{\prime}$ (see Figure 3 ).

Let $m^{*}$ be a meridian of $K=t_{1}^{u} \cup t_{2}^{u}$ in $\partial \eta\left(K ; V_{1}^{\prime}\right)$ and $\ell^{*}$ a longitude of $\partial \eta\left(K ; V_{1}^{\prime}\right)$ such that $\ell^{\prime} \cup \ell^{*}$ bounds a spanning annulus in $\operatorname{cl}\left(V_{1}^{\prime} \backslash \eta\left(K ; V_{1}^{\prime}\right)\right)$. Note that $m^{*}$ and 


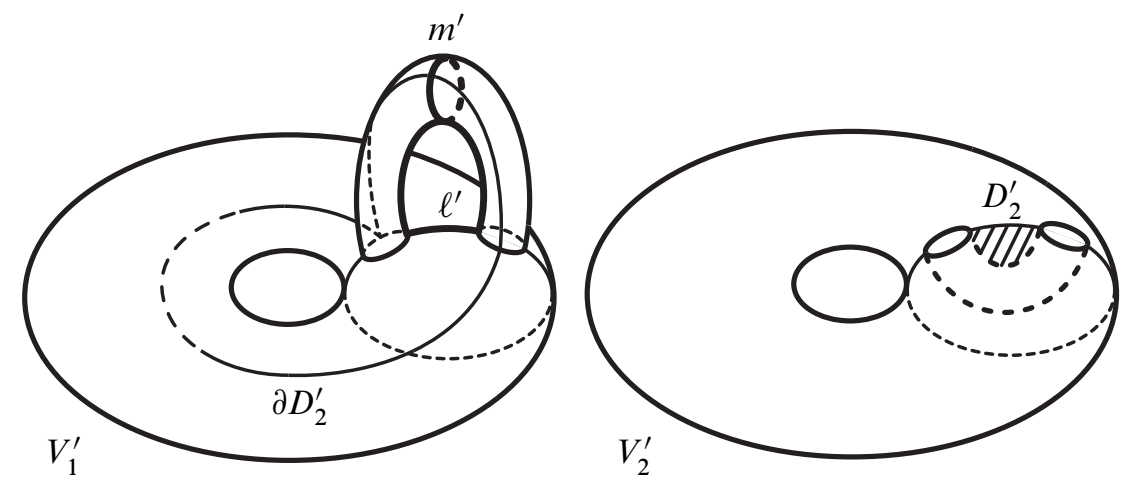

Figure 3

$\ell^{*}$ are oriented as illustrated in Figure 1. Let $r$ and $s$ be coprime integers, and let $V_{1}^{\prime \prime}$ be a genus two handlebody obtained from $\operatorname{cl}\left(V_{1}^{\prime} \backslash \eta\left(K ; V_{1}^{\prime}\right)\right)$ by attaching a solid torus $\bar{V}$ so that a meridian of $\bar{V}$ is identified with a loop represented by $r\left[m^{*}\right]+s\left[\ell^{*}\right]$. Set $M^{\prime}=V_{1}^{\prime \prime} \cup_{S^{\prime}} V_{2}^{\prime}$. Then we say that $M^{\prime}$ is obtained by $(r / s)^{*}$-surgery on $K$. If $r / s$ is an integer, $(r / s)^{*}$-surgery is called an integral surgery.

Definition 2.3 Let $p$ and $q$ be a coprime pair of positive integers. Let $\left\{s_{j}\right\}_{1 \leq j \leq p}$ be the finite sequence such that $0 \leq s_{j}<p$ and $s_{j} \equiv q \cdot j(\bmod p)$. We call such a sequence the basic sequence for $(p, q)$. For an integer $k$ with $0<k<p, \Psi_{p, q}(k)$ denotes the integer $j$ with $s_{j}=k$, and $\Phi_{p, q}(k)$ denotes the number of elements of the following set (possibly empty set):

$$
\left\{s_{j} \mid 1 \leq j<\Psi_{p, q}(k), s_{j}<k\right\} .
$$

Example 2.4 Set $p=22$ and $q=3$. Then we have the basic sequence

$$
\left\{s_{j}\right\}_{1 \leq j \leq 22}: 3,6,9,12,15,18,21,2,5,8,11,14,17,20,1,4,7,10,13,16,19,0 .
$$

Hence we see that $\Psi_{22,3}(5)=9$ and $\Phi_{22,3}(5)=2$.

Remark 2.5 When one follows $\partial D_{2}$ from $P_{0}$ in the positive direction of $\ell$ in Figure 2, $\partial D_{2}$ intersects $\partial D_{1}$ in the following order:

$$
P_{0} \rightarrow P_{s_{1}} \rightarrow P_{s_{2}} \rightarrow \ldots \rightarrow P_{s_{p-1}} \rightarrow P_{0}
$$

Then $\Psi_{p, q}(u)$ represents the number of intersection points between $t_{2}^{\prime}{ }_{2}$ and a parallel copy of $\partial D_{1}$ in $\partial V_{1}$, and $\Phi_{p, q}(u)$ represents the number of intersection points between $t_{1}^{\prime}{ }_{1}^{u}$ and the interior of $t_{2}^{\prime u}$. 
By Remark 2.5, we have the following.

Observation $2.6\left(\operatorname{cf}\left[16\right.\right.$, Theorem 1.3]) For $K=K(L(p, q) ; u)$, if $\Phi_{p, q}(u)=$ $0, u-1$ or $\Psi_{p, q}(u)-1$, then $K$ is isotopic onto $\partial V_{1}\left(=\partial V_{2}\right)$.

In [17], we gave a necessary condition for $K(L(p, q) ; u)$ to admit an integral $S^{3}-$ surgery.

Theorem 2.7 [17, Theorem 2.5] Let $p$ and $q$ be coprime integers with $0<q<p$ and $u$ be an integer with $1 \leq u \leq p-1$. If $K(L(p, q) ; u)$ admits an integral $S^{3}$-surgery, then the surgery is either $0^{*}$ or $1^{*}$-surgery. Moreover, one of the following holds.

(1) $p \cdot \Phi_{p, q}(u)-u \cdot \Psi_{p, q}(u)= \pm 1$. In this case, the surgery is $0^{*}$-surgery.

(2) $p \cdot \Phi_{p, q}(u)-u \cdot \Psi_{p, q}(u)= \pm 1-p$. In this case, the surgery is $1^{*}$-surgery.

The converse of Theorem 2.7 does not hold. For example, though $K(L(22,3) ; 5)$ satisfies the conclusion (1) of Theorem 2.7 , its $0^{*}$-surgery yields a homology sphere other than $S^{3}$. This is confirmed by calculating its fundamental group (cf Section 5). In this paper, we first give an improved necessary condition for $K(L(p, q) ; u)$ to admit an integral $S^{3}$-surgery.

Definition 2.8 Let $p$ and $q$ be coprime integers with $0<q<p$ and $\left\{u_{j}\right\}_{1 \leq j \leq p}$ the basic sequence for $(p, q)$. Let $u$ be an integer with $1 \leq u \leq p-1$. The basic sequence for $(p, q)$ admits $w$-property for $u$ if there does not exist a quadruplet of integers $\{i, j, k, l\}$ which satisfy the following:

(1) $1 \leq i, j, k, l<p$,

(2) $\Psi_{p, q}(i), \Psi_{p, q}(i+1)<\Psi_{p, q}(u)$,

(3) $\Psi_{p, q}(j), \Psi_{p, q}(j+1)>\Psi_{p, q}(u)$,

(4) $\Psi_{p, q}(k)<\Psi_{p, q}(u)<\Psi_{p, q}(k+1)$,

(5) $\Psi_{p, q}(l)>\Psi_{p, q}(u)>\Psi_{p, q}(l+1)$.

Theorem 2.9 Let $p$ and $q$ be coprime integers with $0<q<p / 2$ and $\left\{u_{j}\right\}_{1 \leq j \leq p}$ the basic sequence for $(p, q)$. Let $u$ be an integer with $1 \leq u \leq p / 2$. If $K(L(p, q) ; u)$ admits an integral $S^{3}$-surgery, then the following holds.

(1) $p \cdot \Phi_{p, q}(u)-u \cdot \Psi_{p, q}(u)= \pm 1$ or $\pm 1-p$.

(2) The basic sequence admits $w$-property for $u$. 
A proof of Theorem 2.9 is given in Section 4. By using Theorem 2.9, we easily check that any integral surgery on $K(L(22,3) ; 5)$ cannot give $S^{3}$ as follows. In Definition 2.8 , set $i=2, j=7, k=3$ and $l=1$. Then its basic sequence does not admit w-property for $u=5$ (cf Example 2.4). This together with the conclusion (2) of Theorem 2.9 implies that any integral surgery on $K(L(22,3) ; 5)$ cannot give $S^{3}$.

Theorem 1.4 follows from Theorems 2.10-2.13.

Theorem 2.10 Suppose that $K(L(p, 1) ; u)$ is not a core knot. $K(L(p, 1) ; u)$ with $p>1$ and $1 \leq u \leq p / 2$ admits an integral $S^{3}$-surgery if and only if $(p, u)=(5,2)$.

Moreover, $K(L(5,1) ; 2)$ is a torus knot.

Theorem 2.11 Suppose that $K(L(p, 2) ; u)$ is not a core knot. $K(L(p, 2) ; u)$ with $p>2$ and $1 \leq u \leq p / 2$ admits an integral $S^{3}$-surgery if and only if $(p, u)=$ $(7,3),(9,4)$ or $(11,3)$.

Moreover, $K(L(p, 2) ; u)$ is a torus knot for each $(p, u)$ above.

Theorem 2.12 Suppose that $K(L(p, 3) ; u)$ is not a core knot. $K(L(p, 3) ; u)$ with $p>3$ and $1 \leq u \leq p / 2$ admits an integral $S^{3}$-surgery if and only if $(p, u)=$ $(7,2),(11,5),(13,4),(13,6),(14,5)$ or $(19,4)$.

Moreover, $K(L(p, 3) ; u)$ is a torus knot for each $(p, u)$ above.

Theorem 2.13 Suppose that $K(L(p, 4) ; u)$ is not a core knot. $K(L(p, 4) ; u)$ with $p>4$ and $1 \leq u \leq p / 2$ admits an integral $S^{3}$-surgery if and only if $(p, u)=$ $(13,3),(15,7),(17,8),(21,5),(29,5)$ or $\left(4 p^{\prime}+6 \pm 1,2\right)\left(p^{\prime}=0,1, \ldots\right)$.

Moreover, $K(L(p, 4) ; u)$ is a torus knot for each $(p, u)$ above.

Proofs of Theorems 2.10-2.13 are given in Section 6.

\section{The wave theorem on Heegaard splittings}

A triplet $\left(V_{1}, V_{2} ; S\right)$ is a genus $g$ Heegaard splitting of a closed orientable 3-manifold $N$ if $V_{i}\left(i=1\right.$ and 2) is a genus $g$ handlebody with $N=V_{1} \cup V_{2}$ and $V_{1} \cap V_{2}=$ $\partial V_{1} \cap \partial V_{2}=S$. The surface $S$ is called a Heegaard surface. A properly embedded disk $D$ in a genus $g$ handlebody $V$ is called a meridian disk of $V$ if a 3-manifold obtained by cutting $V$ along $D$ is a genus $g-1$ handlebody. The boundary of a meridian disk of $V$ is called a meridian of $V$. A collection of mutually disjoint $g$ meridians 
$\left\{x_{1}, \ldots, x_{g}\right\}$ of $V$ is called a complete meridian system of $V$ if $\left\{x_{1}, \ldots, x_{g}\right\}$ bounds mutually disjoint meridian disks of $V$ which cuts $V$ into a 3-ball.

Let $\left(V_{1}, V_{2} ; S\right)$ be a genus two Heegaard splitting of $S^{3}$. Let $\left\{x_{1}, x_{2}\right\}$ and $\left\{y_{1}, y_{2}\right\}$ be complete meridian systems of $V_{1}$ and $V_{2}$ respectively. We call $\left(S ;\left\{x_{1}, x_{2}\right\},\left\{y_{1}, y_{2}\right\}\right)$ a Heegaard diagram. If $x_{1}, x_{2}, y_{1}$ and $y_{2}$ are isotoped on $S$ so that the number of their intersection points are minimal, then we call $\left(S ;\left\{x_{1}, x_{2}\right\},\left\{y_{1}, y_{2}\right\}\right)$ a normalized Heegaard diagram. If $\left|x_{1} \cap y_{1}\right|=1,\left|x_{2} \cap y_{2}\right|=1, x_{2} \cap y_{1}=\varnothing$ and $x_{1} \cap y_{2}=\varnothing$, then the Heegaard diagram is said to be standard. Let $\Sigma_{x}\left(\Sigma_{y}\right.$ resp.) be the four holed 2-sphere obtained by cutting $S$ along $x_{1}$ and $x_{2}$ ( $y_{1}$ and $y_{2}$ resp.), and let $x_{i}^{+}$and $x_{i}^{-}\left(y_{i}^{+}\right.$and $y_{i}^{-}$resp.) $(i=1,2)$ be the copies of $x_{i}\left(y_{i}\right.$ resp. $)$ in $\Sigma_{x}\left(\Sigma_{y}\right.$ resp.).

A wave $w$ associated with $x_{i}(i=1$ or 2$)$ is a properly embedded arc in $\Sigma_{x}$ such that $w$ is disjoint from $\left(y_{1} \cup y_{2}\right) \cap \Sigma_{x}, w$ joins $x_{i}^{+}$or $x_{i}^{-}$to itself and $w$ does not cut off a disk from $\Sigma_{x}$. Similarly, a wave $w$ associated with $y_{i}(i=1$ or 2$)$ is a properly embedded arc in $\Sigma_{y}$ such that $w$ is disjoint from $\left(x_{1} \cup x_{2}\right) \cap \Sigma_{y}, w$ joins $y_{i}^{+}$or $y_{i}^{-}$to itself and $w$ does not cut off a disk from $\Sigma_{y}$. A Heegaard diagram $\left(S ;\left\{x_{1}, x_{2}\right\},\left\{y_{1}, y_{2}\right\}\right)$ contains a wave if there is a wave associated with $x_{i}(i=1$ or 2) or $y_{i}(i=1$ or 2$)$. The following, so-called wave theorem, was proved by Homma, Ochiai and Takahashi [10].

Theorem 3.1 [10, Main Theorem] Any normalized genus two Heegaard diagram of $S^{3}$ is standard, or contains a wave.

\section{Proof of Theorem 2.9}
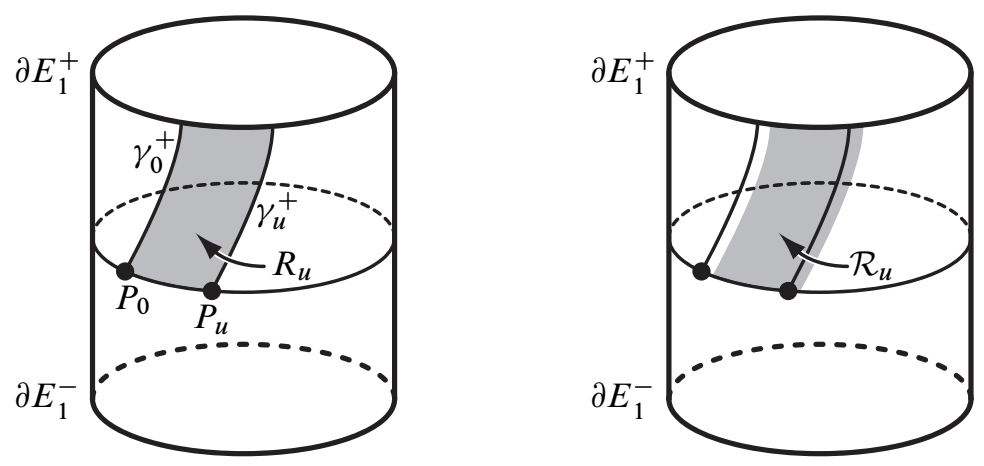

Figure 4 
In this section, we prove Theorem 2.9. In avoiding needless confusion, we shall use the same notation as in Section 2. Recall that $V_{i}(i=1,2)$ give a genus one Heegaard splitting of $L(p, q)$ and that $D_{i}$ 's are meridian disks of $V_{i}$ 's with $K(L(p, q) ; u)=$ $\left(t_{1}^{u} \cup t_{2}^{u}\right) \subset\left(D_{1} \cup D_{2}\right)$. Recall also that $t_{1}^{\prime u}\left(t_{2}^{\prime}{ }_{2}^{u}\right.$ resp.) is a projection of $t_{1}^{u}$ ( $t_{2}^{u}$ resp.) whose initial point is $P_{0}$ and whose endpoint is $P_{u}$ passing in the positive direction of $m$ ( $\ell$ resp.). Let $E_{i}(i=1,2)$ be parallel copies of $D_{i}$, and let $A_{1}$ be the annulus obtained by cutting $\partial V_{1}$ along $\partial E_{1}$. Resulting boundary components are labeled $\partial E_{1}^{ \pm}$so that the orientation of a longitude $\ell$ of $V_{1}$ in $A$ corresponds to the direction toward $\partial E_{1}^{+}$and away from $\partial E_{1}^{-}$. For an integer $j$ with $0 \leq j \leq p-1$, let $\gamma_{j}$ be the arc-component of $\partial D_{2} \cap A_{1}$ passing the point $P_{j}$, and let $\gamma_{j}^{ \pm}$be the subarc of $\gamma_{j}$ joining $P_{j}$ to $\partial E_{1}^{ \pm}$. Let $R_{u}$ be the rectangle obtained by cutting $A_{1}$ along $\gamma_{0}^{+} \cup t^{\prime}{ }_{1}^{u} \cup \gamma_{u}^{+}$. A basic domain $\mathcal{R}_{u}$ means a rectangle obtained by sliding $R_{u}$ slightly in the positive direction of $\partial D_{1}$ (cf Figure 4). Note that $\mathcal{R}_{u}$ contains $\gamma_{j}^{+}$if $1 \leq j \leq u$, and $\mathcal{R}_{u}$ is disjoint from $\gamma_{j}^{+}$otherwise.

Proof of Theorem 2.9 The conclusion (1) of Theorem 2.9 holds by Theorem 2.7. Hence it is enough to prove that the conclusion (2) holds under the assumption and the conclusion (1) of Theorem 2.9.

Note that $\left(V_{1}^{\prime}, V_{2}^{\prime} ; S^{\prime}\right)$ is a genus two Heegaard splitting of $L(p, q)$. Let $D_{2}^{\prime \prime}$ be the component of $D_{2} \cap V_{2}^{\prime}$ other than $D_{2}^{\prime}$. Then $\left\{\partial D_{2}^{\prime}, \partial D_{2}^{\prime \prime}\right\}$ is a complete meridian system of $V_{2}^{\prime}$. Assuming that $K=K(L(p, q) ; u)$ admits an integral $S^{3}$-surgery, it follows from Theorem 2.7 that the surgery is $0^{*}$ or $1^{*}$-surgery. We suppose that the basic sequence for $(p, q)$ does not admit w-property for $u$ to obtain a contradiction as follows.

Case $10^{*}$-surgery on $K$ yields $S^{3}$.

Recall that the exterior of $V_{2}^{\prime}$ in $S^{3}$ is a genus two handlebody denoted by $V_{1}^{\prime \prime}$. Then $\ell^{\prime}$ bounds a meridian disk, say $D_{\ell^{\prime}}$, of $V_{1}^{\prime \prime}$. Let $E_{1}^{\prime}$ be a disk obtained by pushing the interior of $E_{1} \cup \mathcal{R}_{u} \cup D_{\ell^{\prime}}^{\prime}$ into the interior of $V_{1}^{\prime \prime}$ slightly, where $\mathcal{R}_{u}$ is a basic domain and $D_{\ell^{\prime}}^{\prime}$ is a parallel copy of $D_{\ell^{\prime}}$ in $V_{1}^{\prime \prime}$. Note that $E_{1}^{\prime}$ is a meridian disk of $V_{1}^{\prime \prime}$ which is not isotopic to $D_{\ell^{\prime}}$. Choosing $D_{\ell^{\prime}}$ appropriately, we may assume that $E_{1}^{\prime}$ is disjoint from $D_{\ell^{\prime}}$. Set $x_{1}=\partial E_{1}^{\prime}, x_{2}=\ell^{\prime}, y_{1}=\partial D_{2}^{\prime}$ and $y_{2}=\partial D_{2}^{\prime \prime}$. Then $\left(S^{\prime} ;\left\{x_{1}, x_{2}\right\},\left\{y_{1}, y_{2}\right\}\right)$ is a normalized Heegaard diagram of $S^{3}$ (cf Figure 5). Let $\Sigma_{x}$ ( $\Sigma_{y}$ resp.) be the four-holed $2-$ sphere obtained by cutting $S$ along $x_{1}$ and $x_{2}$ ( $y_{1}$ and $y_{2}$ resp.), and let $x_{i}^{+}$and $x_{i}^{-}\left(y_{i}^{+}\right.$and $y_{i}^{-}$resp.) $(i=1,2)$ be the copies of $x_{i}\left(y_{i}\right.$ resp.) in $\Sigma_{x}\left(\Sigma_{y}\right.$ resp.).

Let $\bar{y}_{1}$ be the arc-component of $y_{1} \cap \Sigma_{x}$ which corresponds to $\gamma_{0}^{+}$(and hence $\gamma_{p-1}^{-}$). Then $\bar{y}_{1}$ joins $x_{1}^{+}$to $x_{1}^{-}$. Let $\bar{y}_{2}\left(\bar{y}_{3}\right.$ resp.) be the arc-component of $y_{2} \cap \Sigma_{x}$ which 


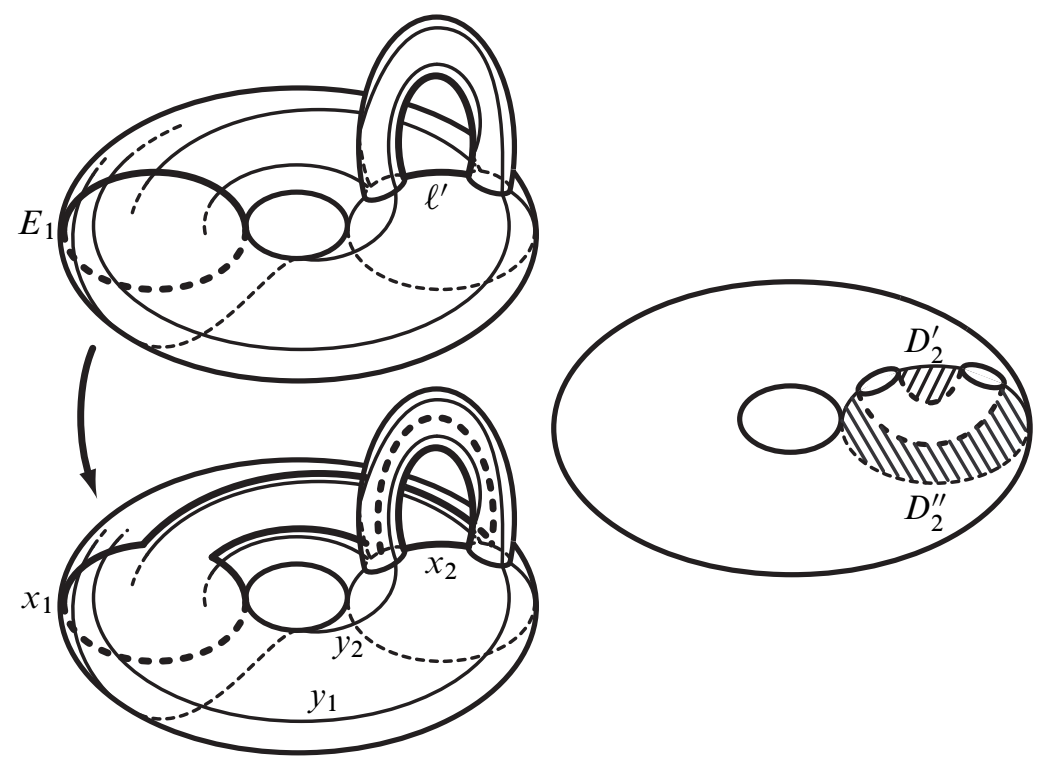

Figure 5

corresponds to $\gamma_{0}^{-}\left(\gamma_{p-1}^{+}\right.$resp.). Then $\bar{y}_{2}$ either joins $x_{1}^{-}$to $x_{2}^{+}$or joins $x_{1}^{-}$to $x_{2}^{-}$, say the latter. Since we assume that $q+1<u<p / 2$, we see that $\bar{y}_{3}$ joins $x_{1}^{+}$to $x_{2}^{+}$. Let $\bar{y}_{4}$ be the arc-component of $\left(y_{1} \cup y_{2}\right) \cap \Sigma_{x}$ which contains $\gamma_{1}^{+}$. Then $\bar{y}_{4}$ joins $P_{1} \subset x_{2}^{+}$to $P_{q+1} \subset x_{2}^{-}$because we assume that $q+1<u<p / 2$. Hence these four arc-components $\bar{y}_{1}, \bar{y}_{2}, \bar{y}_{3}$ and $\bar{y}_{4}$ imply that there are no waves associated with $x_{i}$ $(i=1,2)$ (cf Figure 6).

Since we suppose that the basic sequence for $(p, q)$ does not admit w-property for $u$, there exists a quadruplet of integers $\{i, j, k, l\}$ which satisfy the conditions of Definition 2.8. By the condition (2) of Definition 2.8, we see that $y_{1}$ contains both $P_{i}$ and $P_{i+1}$. Note that $P_{i}$ and $P_{i+1}$ lies on $x_{1}$ ( $x_{2}$ resp.) if $i>u(i<u$ resp.). Let $\bar{x}_{1}$ be the subarc of $x_{1}$ or $x_{2}$ such that $\bar{x}_{1}$ joins $P_{i}$ to $P_{i+1}$ and that the interior of $\bar{x}_{1}$ is disjoint from $y_{1} \cup y_{2}$. Then $\bar{x}_{1}$ joins $y_{1}^{+}$to $y_{1}^{-}$in $\Sigma_{y}$. By the condition (3) of Definition 2.8, we see that $y_{2}$ contains both $P_{j}$ and $P_{j+1}$. Let $\bar{x}_{2}$ be the subarc of $x_{1}$ or $x_{2}$ such that $\bar{x}_{2}$ joins $P_{j}$ to $P_{j+1}$ and that the interior of $\bar{x}_{2}$ is disjoint from $y_{1} \cup y_{2}$. Then $\bar{x}_{2}$ joins $y_{2}^{+}$to $y_{2}^{-}$in $\Sigma_{y}$. By the condition (4) of Definition 2.8, we see that $y_{1}$ contains $P_{k}$ and $y_{2}$ contains $P_{k+1}$. Let $\bar{x}_{3}$ be the subarc of $x_{1}$ or $x_{2}$ such that $\bar{x}_{3}$ joins $P_{k}$ to $P_{k+1}$ and that the interior of $\bar{x}_{3}$ is disjoint from $y_{1} \cup y_{2}$. Then $\bar{x}_{3}$ either joins $y_{1}^{+}$to $y_{2}^{-}$or joins $y_{1}^{-}$to $y_{2}^{+}$, say the former, in $\Sigma_{y}$. By condition (4) 

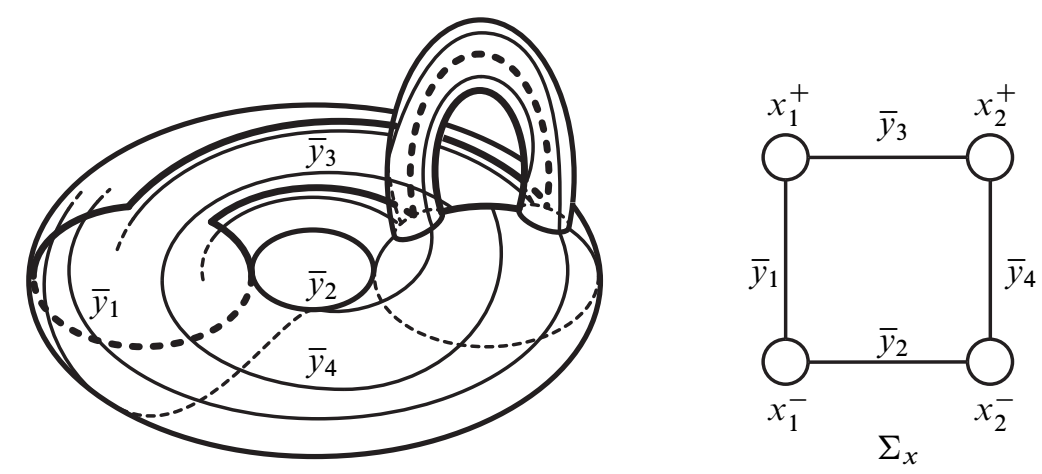

Figure 6

of Definition 2.8, we see that $y_{2}$ contains $P_{l}$ and $y_{1}$ contains $P_{l+1}$. Let $\bar{x}_{4}$ be the subarc of $x_{1}$ or $x_{2}$ such that $\bar{x}_{4}$ joins $P_{l}$ to $P_{l+1}$ and that the interior of $\bar{x}_{4}$ is disjoint from $y_{1} \cup y_{2}$. Then $\bar{x}_{4}$ joins $y_{1}^{-}$to $y_{2}^{+}$in $\Sigma_{y}$. We see that these four arc-components $\bar{x}_{1}, \bar{x}_{2}, \bar{x}_{3}$ and $\bar{x}_{4}$ imply that there are no waves associated with $y_{i}(i=1,2)$. By Theorem 3.1, this contradicts that $\left(S^{\prime} ;\left\{x_{1}, x_{2}\right\},\left\{y_{1}, y_{2}\right\}\right)$ is a normalized Heegaard diagram of $S^{3}$ and therefore the conclusion (2) of Theorem 2.9 holds.
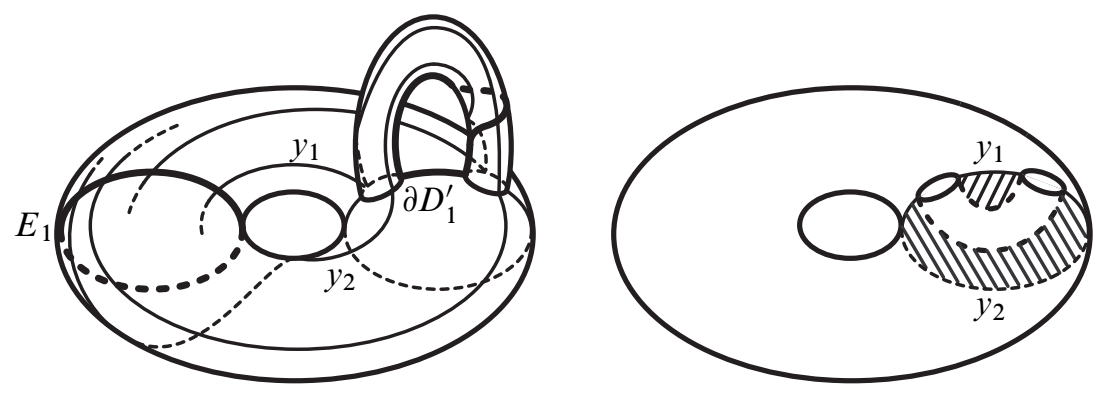

Figure 7

Case $21^{*}$-surgery on $K$ yields $S^{3}$.

Recall that $m^{\prime}$ is as in Section 2 (cf Figure 3). Let $D_{1}^{\prime}$ be a meridian disk of a solid torus obtained by cutting $V_{1}^{\prime \prime}$ along $E_{1}$ such that $\partial D_{1}^{\prime} \cap E\left(m^{\prime} ; \partial V_{1}^{\prime \prime}\right)$ is identified with $\ell^{\prime} \cap E\left(m^{\prime} ; \partial V_{1}^{\prime \prime}\right)$ (cf Figure 7). Let $E_{1}^{\prime}$ be a disk obtained by pushing the interior of $E_{1} \cup \mathcal{R}_{u} \cup D_{1}^{\prime \prime}$ into the interior of $V_{1}^{\prime \prime}$ slightly, where $\mathcal{R}_{u}$ is a basic domain and 
$D_{1}^{\prime \prime}$ is a parallel copy of $D_{1}^{\prime}$ in $V_{1}^{\prime \prime}$. Note that $E_{1}^{\prime}$ is a meridian disk of $V_{1}^{\prime \prime}$ which is not isotopic to $D_{1}^{\prime}$. Choosing $D_{1}^{\prime \prime}$ appropriately, we may assume that $E_{1}^{\prime}$ is disjoint from $D_{1}^{\prime}$. Set $x_{1}=\partial E_{1}^{\prime}, x_{2}=\partial D_{1}^{\prime}$ and let $y_{1}$ and $y_{2}$ be as in Case 1 above. Then $\left(S^{\prime} ;\left\{x_{1}, x_{2}\right\},\left\{y_{1}, y_{2}\right\}\right)$ is a normalized Heegaard diagram of $S^{3}$. By an argument similar to that in Case 1, we also see that this diagram contains no waves because we suppose the basic sequence for $(p, q)$ does not admit w-property for $u$. Therefore the conclusion (2) of Theorem 2.9 holds.

\section{Presentation of fundamental groups}

To avoid needless confusion, we shall also use the same notation in Section 2.

Proposition 5.1 Set $K=K(L(p, q) ; u)$ and let $\left\{s_{j}\right\}_{1 \leq j \leq p}$ be the basic sequence for $(p, q)$. Let $N^{\prime}$ be the 3-manifold obtained by $r^{*}$-surgery on $K$, where $r$ be an integer. Then we have

$$
\pi_{1}\left(N^{\prime}\right) \cong\left\langle a, b \mid \prod_{j=1}^{\Psi_{p, q}(u)} W_{1}(j)=1, \prod_{j=1}^{p} W_{2}(j)=1\right\rangle
$$

where $\quad W_{1}(j)=\left\{\begin{array}{cl}a & \text { if } s_{j}>u \\ a b^{r} & \text { if } s_{j}=u \\ a b & \text { otherwise }\end{array} \quad\right.$ and $\quad W_{2}(j)=\left\{\begin{array}{cc}a & \text { if } s_{j} \geq u \\ a b & \text { otherwise }\end{array}\right.$

Proof Recall that $\left(V_{1}^{\prime \prime}, V_{2}^{\prime} ; S^{\prime}\right)$ be a genus two Heegaard splitting of $N^{\prime}$. Since $N^{\prime}$ is obtained by an integral surgery on $K$, we see that $a:=\ell$ and $b:=m^{\prime}$ are free generators of $\pi_{1}\left(V_{1}^{\prime \prime}\right)$ (cf Figure 8). Let $E_{i}(i=1,2)$ be parallel copies of $D_{i}$ in $V_{i}$. In particular, we may assume that $\partial E_{2}$ in $\partial V_{1}$ is obtained by sliding $\partial D_{2}$ slightly in the positive direction of $\partial D_{1}$. Then it follows from Van Kampen's theorem that

$$
\pi_{1}\left(N^{\prime}\right) \cong\left\langle a, b \mid \partial D_{2}^{\prime}=1, \partial E_{2}=1\right\rangle .
$$

Let $D_{1}^{\prime}$ be a meridian disk of a solid torus obtained by cutting $V_{1}^{\prime \prime}$ along $E_{1}$ such that $\partial D_{1}^{\prime} \cap E\left(m^{\prime} ; \partial V_{1}^{\prime \prime}\right)$ is identified with $\ell^{\prime} \cap E\left(m^{\prime} ; \partial V_{1}^{\prime \prime}\right)$. Since $N^{\prime}$ is obtained by $r^{*}$-surgery on $K, \partial D_{1}^{\prime}$ rounds $|r|$ times in the positive or negative direction of $m^{\prime}$ in $N\left(m^{\prime} ; \partial V_{1}^{\prime \prime}\right)$. We remark that its direction depends on the sign of $r$. By Remark 2.5, when one follows $\partial D_{2}^{\prime}$ from a neighborhood of $P_{0}$ in the positive direction of $\ell, \partial D_{2}^{\prime}$ intersects $\partial D_{1}^{\prime}$ in the following order:

$$
\left(P_{0} \rightarrow\right) P_{S_{1}} \rightarrow P_{S_{2}} \rightarrow \ldots \rightarrow P_{\Psi_{p, q}(u)-1}\left(\rightarrow P_{\Psi_{p, q}(u)}\right) .
$$




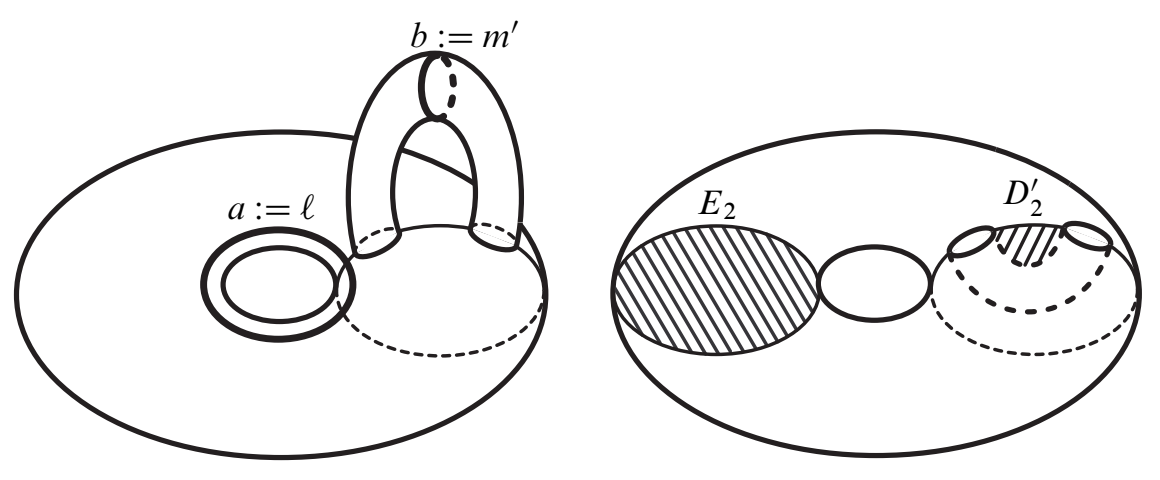

Figure 8

We note that $\partial D_{2}^{\prime}$ intersects $\partial E_{1}$ just one time on the way from $P_{s_{j}}$ to $P_{s_{j}+1}$. If $s_{j}<u$, then $\partial D_{2}^{\prime}$ intersects $\partial D_{1}^{\prime}$ at the point $P_{s_{j}}$. In the annulus $\partial \eta\left(t_{2}^{u} ; V_{2}\right), \partial D_{2}^{\prime}$ is disjoint from $\partial E_{1}$ and intersects $\partial D_{1}^{\prime}$ in $|r|$ points. Considering the sign of $r$, we see that $\partial D_{2}^{\prime}$ is presented by

$$
\prod_{j=1}^{\Psi_{p, q}(u)} W_{1}(j)
$$

Similarly, we also see that $\partial E_{2}$ is presented by $\prod_{j=1}^{p} W_{2}(j)$.

Corollary 5.2 Set $K=K(L(p, q) ; u)$ and let $\left\{s_{j}\right\}_{1 \leq j \leq p}$ be the basic sequence for $(p, q)$. Then we have

$$
\pi_{1}(E(K ; L(p, q))) \cong\left\langle b, c \mid \prod_{j=1}^{p} W_{3}(j)=1\right\rangle,
$$

where

$$
W_{3}(j)= \begin{cases}c b & \text { if } \Psi_{p, q}\left(s_{j}\right)<\Psi_{p, q}(u) \\ c & \text { otherwise }\end{cases}
$$

Proof We use the same notation as in the proof of Proposition 5.1. Particularly, we here regard $b=m^{\prime}$ as a free generator of $V_{2}^{\prime}$. Note that $b$ intersects $\partial D_{2}^{\prime}$ transversely in a single point and is disjoint from $\partial E_{2}$ Let $c$ be a simple loop in $V_{2}^{\prime}$ which intersects $\partial E_{2}$ transversely in a single point and is disjoint from $\partial D_{2}^{\prime}$. Then $c$ is also a free generator of $V_{2}^{\prime \prime}$ other than $b$. It follows from Van Kampen's theorem that

$$
\pi_{1}(E(K ; L(p, q))) \cong\left\langle b, c \mid \partial E_{1}=1\right\rangle .
$$


Recall that $E_{1}$ is a parallel copy of $D_{1}$. Let $P_{i}^{\prime} \subset E_{1}(i=0,1, \ldots, p-1)$ be parallel copies of $P_{i} \subset \partial D_{1}$. When we follow $\partial E_{1}$ in the positive direction of $\partial E_{1}$, we see that $\partial E_{1}$ intersects $\partial E_{2}$ just one time on the way from $P_{j}^{\prime}$ to $P_{j+1}^{\prime}$. If $\Psi_{p, q}\left(s_{j}\right)<\Psi_{p, q}(u)$, then $\partial E_{1}$ intersects $\partial D_{1}^{\prime}$ at the point $P_{s_{j}}^{\prime}$. Hence we see that $\partial E_{1}$ is presented by $\prod_{j=1}^{p} W_{3}(j)$.

Example 5.3 Set $K=K(L(22,3) ; 5)$ and let $N^{\prime}$ be the 3 -manifold obtained by $0^{*}$-surgery on $K$. Recall that the basic sequence for $(22,3)$ is written in Example 2.4. Then Proposition 5.1 indicates that

$$
\pi_{1}\left(N^{\prime}\right) \cong\left\langle a, b \mid a^{2} b a^{7} b=1, a^{6} b a b a^{6} b=1\right\rangle .
$$

Hence we have

$$
\begin{aligned}
\pi_{1}\left(N^{\prime}\right) & \cong\left\langle a, \bar{b} \mid a \bar{b} a^{-4} \bar{b}=1, a^{-5} \bar{b}^{3}=1\right\rangle \quad\left(\bar{b}:=a^{6} b\right) \\
& \cong\left\langle a, \bar{b} \mid(a \bar{b})^{2} a^{-5}=1, a^{-5} \bar{b}^{3}=1\right\rangle \\
& \cong\left\langle a, \bar{b} \mid a^{5}=(a \bar{b})^{2}=\bar{b}^{3}\right\rangle .
\end{aligned}
$$

This implies that $\pi_{1}\left(N^{\prime}\right)$ is isomorphic to the binary icosahedral group and hence $\pi_{1}\left(N^{\prime}\right)$ is nontrivial.

The following is easily obtained by using Corollary 5.2. Calculation is left for the reader.

Observation 5.4 The following will be all knots represented by $K(L(p, q) ; u)$ with $0<q<5$ each of which admits an integral $S^{3}$-surgery and is not a core knot. (We assume that $0<q<p / 2$ and $0<u \leq p / 2$.)

(1) If $K=K(L(5,1) ; 2)$, then $\pi_{1}(E(K ; L(5,1))) \cong\left\langle x, y \mid x^{3}=y^{2}\right\rangle$.

(2) If $K=K(L(7,2) ; 3)$, then $\pi_{1}(E(K ; L(7,2))) \cong\left\langle x, y \mid x^{3}=y^{2}\right\rangle$.

(3) If $K=K(L(9,2) ; 4)$, then $\pi_{1}(E(K ; L(9,2))) \cong\left\langle x, y \mid x^{5}=y^{2}\right\rangle$.

(4) If $K=K(L(11,2) ; 3)$, then $\pi_{1}(E(K ; L(11,2))) \cong\left\langle x, y \mid x^{4}=y^{3}\right\rangle$.

(5) If $K=K(L(7,3) ; 2)$, then $\pi_{1}(E(K ; L(7,3))) \cong\left\langle x, y \mid x^{3}=y^{2}\right\rangle$.

(6) If $K=K(L(11,3) ; 5)$, then $\pi_{1}(E(K ; L(11,3))) \cong\left\langle x, y \mid x^{5}=y^{2}\right\rangle$.

(7) If $K=K\left(L(13,3)\right.$; 4), then $\pi_{1}(E(K ; L(13,3))) \cong\left\langle x, y \mid x^{4}=y^{3}\right\rangle$.

(8) If $K=K(L(13,3) ; 6)$, then $\pi_{1}(E(K ; L(13,3))) \cong\left\langle x, y \mid x^{7}=y^{2}\right\rangle$.

(9) If $K=K(L(14,3) ; 5)$, then $\pi_{1}(E(K ; L(14,3))) \cong\left\langle x, y \mid x^{5}=y^{3}\right\rangle$.

(10) If $K=K(L(19,3) ; 4)$, then $\pi_{1}(E(K ; L(19,3))) \cong\left\langle x, y \mid x^{5}=y^{4}\right\rangle$. 
(11) If $K=K(L(13,4) ; 3)$, then $\pi_{1}(E(K ; L(13,4))) \cong\left\langle x, y \mid x^{4}=y^{3}\right\rangle$.

(12) If $K=K(L(15,4) ; 7)$, then $\pi_{1}(E(K ; L(15,4))) \cong\left\langle x, y \mid x^{7}=y^{2}\right\rangle$.

(13) If $K=K(L(17,4) ; 8)$, then $\pi_{1}(E(K ; L(17,4))) \cong\left\langle x, y \mid x^{9}=y^{2}\right\rangle$.

(14) If $K=K(L(21,4) ; 5)$, then $\pi_{1}(E(K ; L(21,4))) \cong\left\langle x, y \mid x^{5}=y^{4}\right\rangle$.

(15) If $K=K(L(29,4) ; 5)$, then $\pi_{1}(E(K ; L(29,4))) \cong\left\langle x, y \mid x^{6}=y^{5}\right\rangle$.

(16) If $K=K\left(L\left(4 p^{\prime}+5,4\right) ; 2\right)$ for a nonnegative integer $p^{\prime}$, then $\pi_{1}\left(E\left(K ; L\left(4 p^{\prime}+5,4\right)\right)\right) \cong\left\langle x, y \mid x^{2 p^{\prime}+3}=y^{2}\right\rangle$.

(17) If $K=K\left(L\left(4 p^{\prime}+7,4\right)\right.$; $)$ for a nonnegative integer $p^{\prime}$, then $\pi_{1}\left(E\left(K ; L\left(4 p^{\prime}+7,4\right)\right)\right) \cong\left\langle x, y \mid x^{2 p^{\prime}+3}=y^{2}\right\rangle$.

\section{Proofs of Theorems 2.10-2.13}

\subsection{Proof of Theorem 2.10}

Lemma 6.1 Suppose that $K(L(p, 1) ; u)$ is not a core knot. If $K(L(p, 1) ; u)$ with $p>1$ and $1 \leq u \leq p / 2$ admits an integral $S^{3}$-surgery, then $(p, u)=(5,2)$.

Moreover, $K(L(5,1) ; 2)$ is a torus knot.

Proof Set $K=K(L(p, 1) ; u)$ with $p>1$. Then the basic sequence for $(p, 1)$ is the following:

$$
\left\{s_{j}\right\}_{1 \leq j \leq p}: 1,2,3, \ldots, p-1,0 .
$$

Hence we see that $\Phi_{p, 1}(u)=u-1$ and $\Psi_{p, 1}(u)=u$. Suppose that $K$ admits an integral $S^{3}$-surgery. Then $K$ satisfies the conclusion (1) of Theorem 2.9. We divide the proof into the following cases.

Case $1 p \cdot \Phi_{p, 1}(u)-u \cdot \Psi_{p, 1}(u)= \pm 1$.

If $u=1$, then $\Psi_{p, 1}(u)=1$. This implies that $K$ is a core knot, a contradiction. Hence $u \neq 1$. Since $\Phi_{p, 1}(u)=u-1$ and $\Psi_{p, 1}(u)=u$, we see that $p=\left(u^{2} \pm 1\right) /(u-1)$. If $p=\left(u^{2}-1\right) /(u-1)$, then $p=u+1$. This also implies that $K$ is a core knot, a contradiction. Hence we see that $p=\left(u^{2}+1\right) /(u-1)$ and hence $p=u+1+2 /(u-1)$. Since $u$ and $p$ are positive integers, we see that $u=2,3$ and hence $p=5$. However, if $(p, u)=(5,3)$, then this contradicts that $1 \leq u \leq p / 2$. Hence we see that $(p, u)=(5,2)$. Since $\Phi_{5,1}(2)=1=u-1$, Since $\Phi_{9,2}(4)=1=\Psi_{9,2}(4)-1$, it follows from Observation 2.6 that $K$ is isotopic to a Heegaard torus. Moreover, it follows from Observation 5.4 that $K(L(5,1) ; 2)$ is not a core knot. Hence $K(L(5,1) ; 2)$ is a torus knot.

Case $2 p \cdot \Phi_{p, 1}(u)-u \cdot \Psi_{p, 1}(u)= \pm 1-p$. 
Since $\Phi_{p, 1}(u)=u-1$ and $\Psi_{p, 1}(u)=u$, we see that $p=u \pm 1 / u$. Since $u$ and $p$ are positive integers, we see that $u=1$ and hence $K$ is a core knot, a contradiction.

Lemma 6.2 For $(p, u)=(5,2), 0^{*}$-surgery on $K=K(L(p, q) ; u)$ is an integral $S^{3}$-surgery.

Proof Let $N^{\prime}$ be the 3 -manifold obtained by $0^{*}$-surgery on $K$. Then Proposition 5.1 indicates that

$$
\pi_{1}\left(N^{\prime}\right) \cong\left\langle a, b \mid a b a=1, a b a^{4} b=1\right\rangle .
$$

Hence we see that $\pi_{1}\left(N^{\prime}\right)$ is trivial by using word reduction (cf [11]). Since Poincaré conjecture is true for the genus two 3 -manifolds ( $\mathrm{cf}[3 ; 4]$ ), we see that $N^{\prime}$ is homeomorphic to $S^{3}$.

\subsection{Proof of Theorem 2.11}

Lemma 6.3 Suppose that $K(L(p, 2) ; u)$ is not a core knot. If $K(L(p, 2) ; u)$ with $p>2$ and $1 \leq u \leq p / 2$ admits an integral $S^{3}$-surgery, then $(p, u)=(7,3),(9,4)$ or $(11,3)$.

Moreover, $K(L(p, 2) ; u)$ is a torus knot for each $(p, u)$ above.

Proof Set $K=K(L(p, 2) ; u)$ with $p>2$. Note that $p \not \equiv 0(\bmod 2)$. Then the basic sequence for $(p, 2)$ is the following:

$$
\left\{s_{j}\right\}_{1 \leq j \leq p}: 2,4, \ldots, p-1,1,3, \ldots, p-2,0 .
$$

In other words,

$$
s_{j}=\left\{\begin{array}{cl}
2 j & \text { if } 1 \leq j \leq(p-1) / 2 \\
2 j-p & \text { if }(p+1) / 2 \leq j \leq p-1 \\
0 & \text { if } j=p .
\end{array}\right.
$$

We divide the proof into the following cases.

Case $1 u \equiv 0(\bmod 2)$.

Set $u=2 t$ for a positive integer $t$. Then we see that $\Phi_{p, 2}(u)=t-1$ and $\Psi_{p, 2}(u)=t$. Suppose that $K$ admits an integral $S^{3}$-surgery.

Case $1.1 p \cdot \Phi_{p, 2}(u)-u \cdot \Psi_{p, 2}(u)= \pm 1$.

If $t=1$, then $\Psi_{p, 2}(u)=1$. This implies that $K$ is a core knot, a contradiction. Hence $t \neq 1$. Since $u=2 t, \Phi_{p, 2}(u)=t-1$ and $\Psi_{p, 2}(u)=t$, we see that $p=$ $2 t+2+(2 \pm 1) /(t-1)$. Since $t$ and $p$ are positive integers, we see that $t=2$, 4 . If 
$t=2$, then $(p, u)=(7,4)$ or $(9,4)$. Since we assume that $1 \leq u \leq p / 2$, we see that $(p, u) \neq(7,4)$ and hence $(p, u)=(9,4)$. Since $\Phi_{9,2}(4)=1=\Psi_{9,2}(4)-1$, it follows from Observation 2.6 that $K$ is isotopic to a Heegaard torus. Moreover, it follows from Observation 5.4 that $K(L(7,2) ; 4)$ is not a core knot. Hence $K(L(7,2) ; 4)$ is a torus knot. If $t=4$, then $(p, u)=(11,8)$. This contradicts that $1 \leq u \leq p / 2$.

Case $1.2 p \cdot \Phi_{p, 1}(u)-u \cdot \Psi_{p, 1}(u)= \pm 1-p$.

Then we see that $p=2 t \pm 1 / t$. Since $p$ is an integer with $p>2$, we see that $t=1$ and hence $K$ is a core knot, a contradiction.

Case $2 u \equiv 1(\bmod 2)$.

Set $u=2 t-1$ for a positive integer $t$. Then we see that $\Phi_{p, 2}(u)=2 t-2$ and $\Psi_{p, 1}(u)=(p-1) / 2+t$. Suppose that $K$ admits an integral $S^{3}$-surgery.

Case $2.1 p \cdot \Phi_{p, 2}(u)-u \cdot \Psi_{p, 2}(u)= \pm 1$.

Then we see that $p=2 t+1+(4 \pm 2)(2 t-3)$. Since $t$ and $p$ are positive integers with $p>2$, we see that $t=2,3$. If $t=2$, then $(p, u)=(7,3)$ or $(11,3)$. In each case, it follows from Observations 2.6 and 5.4 that $K$ is isotopic to a Heegaard torus and is not a core knot. Hence $K$ is a torus knot. If $t=3$, then this contradicts that $1 \leq u \leq p / 2$.

Case $2.2 p \cdot \Phi_{p, 1}(u)-u \cdot \Psi_{p, 1}(u)= \pm 1-p$.

Then we see that $p=2 t-1 \pm 2 /(2 t-1)$. Since $t$ and $p$ are positive integers, we see that $(p, u)=(3,1)$ and hence $K$ is a core knot, a contradiction.

Lemma 6.4 For each $(p, u)=(7,3),(9,4)$ and $(11,3), 0^{*}$-surgery on the knot $K=K(L(p, 2) ; u)$ is an integral $S^{3}$-surgery.

Proof Let $N^{\prime}$ be the 3-manifold obtained by $0^{*}$-surgery on $K$. Then Proposition 5.1 indicates the following.

(1) If $(p, u)=(7,3)$, then

$$
\pi_{1}\left(N^{\prime}\right) \cong\left\langle a, b \mid a b a^{3} b a=1, a b a^{3} b a^{3} b=1\right\rangle .
$$

(2) If $(p, u)=(9,4)$, then

$$
\pi_{1}\left(N^{\prime}\right) \cong\left\langle a, b \mid a b a=1, a b a^{4} b a b a^{3} b=1\right\rangle .
$$

(3) If $(p, u)=(11,3)$, then

$$
\pi_{1}\left(N^{\prime}\right) \cong\left\langle a, b \mid a b a^{5} b a=1, a b a^{5} b a^{5} b=1\right\rangle .
$$


In each case, we see that $\pi_{1}\left(N^{\prime}\right)$ is trivial by using word reduction and hence $N^{\prime}$ is homeomorphic to $S^{3}$.

\subsection{Proof of Theorem 2.12}

Lemma 6.5 Suppose that $K(L(p, 3) ; u)$ is not a core knot. If $K(L(p, 3) ; u)$ with $p>3$ and $1 \leq u \leq p / 2$ admits an integral $S^{3}$-surgery, then $(p, u)=(7,2),(11,5)$, $(13,4),(13,6),(14,5)$ or $(19,4)$.

Moreover, $K(L(p, 3) ; u)$ is a torus knot for each $(p, u)$ above.

Proof Set $K=K(L(p, 3) ; u)$ with $p>3$. Note that $p \not \equiv 0(\bmod 3)$.

Case A $p \equiv 1(\bmod 3)$.

Then the basic sequence for $(p, 3)$ is the following:

$$
\left\{s_{j}\right\}_{1 \leq j \leq p}: 3,6, \ldots, p-1,2,5, \ldots, p-2,1,4, \ldots, p-3,0 .
$$

In other words,

$$
s_{j}=\left\{\begin{array}{cl}
3 j & \text { if } 1 \leq j \leq(p-1) / 3 \\
3 j-p & \text { if }(p+2) / 3 \leq j \leq(2 p-2) / 3 \\
3 j-2 p & \text { if }(2 p+1) / 3 \leq j \leq p-1 \\
0 & \text { if } j=p .
\end{array}\right.
$$

We divide the proof into the following cases.

Case A.1 $u \equiv 0(\bmod 3)$.

Set $u=3 t$ for a positive integer $t$. Then we see that $\Phi_{p, 3}(u)=t-1$ and $\Psi_{p, 3}(u)=t$. Suppose that $K$ admits an integral $S^{3}$-surgery.

Case A.1.1 $p \cdot \Phi_{p, 3}(u)-u \cdot \Psi_{p, 3}(u)= \pm 1$.

If $t=1$, then $\Psi_{p, 3}(u)=1$. This implies that $K$ is a core knot, a contradiction. Hence $t \neq 1$. Hence we see that $p=3 t+3+(3 \pm 1) /(t-1)$. Since $t$ and $p$ are positive integers, we see that $t=2,3,5$. If $t=2$, then $(p, u)=(13,6)$ because $p \equiv 1(\bmod 3)$. Hence it follows from Observations 2.6 and 5.4 that $K$ is isotopic to a Heegaard torus and is not a core knot. Hence $K$ is a torus knot. If $t=4$ or 5 , then this contradicts that $p \equiv 1(\bmod 3)$ or $1 \leq u \leq p / 2$.

Case A.1.2 $p \cdot \Phi_{p, 3}(u)-u \cdot \Psi_{p, 3}(u)= \pm 1-p$.

Then we see that $p=3 t \pm 1 / t$. Since $t$ and $p$ are positive integers with $p>3$, we see that $t=1$ and hence $K$ is a core knot, a contradiction. 
Case A.2 $u \equiv 1(\bmod 3)$.

Set $u=3 t-2$ for a positive integer $t$. Then we see that $\Phi_{p, 3}(u)=3 t-3$ and $\Psi_{p, 3}(u)=(2 p-2) / 3+t$. Suppose that $K$ admits an integral $S^{3}$-surgery.

Case A.2.1 $p \cdot \Phi_{p, 3}(u)-u \cdot \Psi_{p, 3}(u)= \pm 1$.

Then we see that $p=3 t+1+(9 \pm 3) /(3 t-5)$. Since $t$ and $p$ are positive integers with $p>3$, we see that $t=2,3$. If $t=2$, then $(p, u)=(13,4)$ or $(19,4)$. In each case, it follows from Observations 2.6 and 5.4 that $K$ is isotopic to a Heegaard torus and is not a core knot. Hence $K$ is a torus knot. If $t=3$, then this contradicts that $1 \leq u \leq p / 2$.

Case A.2.2 $p \cdot \Phi_{p, 3}(u)-u \cdot \Psi_{p, 3}(u)= \pm 1-p$.

Then we see that $p=3 t-2 \pm 3 /(3 t-2)$. Since $t$ and $p$ are positive integers, we see that $t=1$ and hence $K$ is a core knot, a contradiction.

Case A.3 $u \equiv 2(\bmod 3)$.

Set $u=3 t-1$ for a positive integer $t$. Then we see that $\Phi_{p, 3}(u)=2 t-2$ and $\Psi_{p, 3}(u)=(p-1) / 3+t$. Suppose that $K$ admits an integral $S^{3}$-surgery.

Case A.3.1 $p \cdot \Phi_{p, 3}(u)-u \cdot \Psi_{p, 3}(u)= \pm 1$.

Then we see that $p=3 t+3+(16 \pm 3) /(3 t-5)$. Since $t$ and $p$ are positive integers, we see that $t=2,6,8$. If $t=2$, then $(p, u)=(22,5)$ or $(28,5)$. In each case, however, the basic sequence for $(p, 3)$ does not admit w-property for $u=5$. This contradicts the conclusion (2) of Theorem 2.9. If $t=6,8$, then this contradicts that $1 \leq u \leq p / 2$.

Case A.3.2 $p \cdot \Phi_{p, 3}(u)-u \cdot \Psi_{p, 3}(u)= \pm 1-p$.

Then we see that $p=3 t+(1 \pm 3) /(3 t-2)$. Since $t$ and $p$ are positive integers with $p>3$, we see that $t=1,2$. If $t=1$, then $(p, u)=(7,2)$. Hence it follows from Observations 2.6 and 5.4 that $K$ is isotopic to a Heegaard torus and is not a core knot. Hence $K$ is a torus knot. If $t=2$, then this contradicts that $1 \leq u \leq p / 2$.

Case B $p \equiv 2(\bmod 3)$.

Then the basic sequence for $(p, 3)$ is the following:

$$
\left\{s_{j}\right\}_{1 \leq j \leq p}: 3,6, \ldots, p-2,1,4, \ldots, p-1,2,5, \ldots, p-3,0 .
$$

In other words,

$$
s_{j}=\left\{\begin{array}{cl}
3 j & \text { if } 1 \leq j \leq(p-2) / 3 \\
3 j-p & \text { if }(p+1) / 3 \leq j \leq(2 p-1) / 3 \\
3 j-2 p & \text { if }(2 p+2) / 3 \leq j \leq p-1 \\
0 & \text { if } j=p
\end{array}\right.
$$


We divide the proof into the following cases.

Case B.1 $u \equiv 0(\bmod 3)$.

Set $u=3 t$ for a positive integer $t$. Then we see that $\Phi_{p, 3}(u)=t-1$ and $\Psi_{p, 3}(u)=t$. By the same argument as in Case A.1, we have $(p, u)=(11,6)$ because $p \equiv 2(\bmod 3)$ and $1 \leq u \leq p / 2$. Hence it follows from Observations 2.6 and 5.4 that $K$ is isotopic to a Heegaard torus and is not a core knot. Hence $K$ is a torus knot.

Case B.2 $u \equiv 1(\bmod 3)$.

Set $u=3 t-2$ for a positive integer $t$. Then we see that $\Phi_{p, 3}(u)=2 t-2$ and $\Psi_{p, 3}(u)=(p-2) / 3+t$. Suppose that $K$ admits an integral $S^{3}$-surgery.

Case B.2.1 $p \cdot \Phi_{p, 3}(u)-u \cdot \Psi_{p, 3}(u)= \pm 1$.

Then we see that $p=3 t+(4 \pm 3) /(3 t-4)$. Since $t$ and $p$ are positive integers with $p>3$, we see that this case is impossible.

Case B.2.2 $p \cdot \Phi_{p, 3}(u)-u \cdot \Psi_{p, 3}(u)= \pm 1-p$.

Then we see that $p=3 t-3+(1 \pm 3) /(3 t-1)$. Since $t$ and $p$ are positive integers with $p>3$, we see that this case is also impossible.

Case B.3 $u \equiv 2(\bmod 3)$.

Set $u=3 t-1$ for a positive integer $t$. Then we see that $\Phi_{p, 3}(u)=3 t-2$ and $\Psi_{p, 3}(u)=(2 p-1) / 3+t$. Suppose that $K$ admits an integral $S^{3}$-surgery.

Case B.3.1 $p \cdot \Phi_{p, 3}(u)-u \cdot \Psi_{p, 3}(u)= \pm 1$.

Then we see that $p=3 t+2+(9 \pm 3) /(3 t-4)$. Since $t$ and $p$ are positive integers, we see that $t=2$ and hence $(p, u)=(11,5)$ or $(14,5)$. In each case, it follows from Observations 2.6 and 5.4 that $K$ is isotopic to a Heegaard torus and is not a core knot. Hence $K$ is a torus knot.

Case B.3.2 $p \cdot \Phi_{p, 3}(u)-u \cdot \Psi_{p, 3}(u)= \pm 1-p$.

Then we see that $p=3 t-1 \pm 3 /(3 t-1)$. This implies that $p$ is not an integer for any positive integer $t$.

Lemma 6.6 For each $(p, u)=(11,5),(13,4),(13,6),(14,5)$ and $(19,4), 0^{*}-$ surgery on $K(L(p, 3) ; u)$ is an integral $S^{3}$-surgery. For $(p, u)=(7,2), 1^{*}$-surgery on $K(L(p, 3) ; u)$ is an integral $S^{3}$-surgery.

Proof Let $N_{r}^{\prime}$ be the 3-manifold obtained by $r^{*}$-surgery on $K(L(p, 3) ; u)$. Then Proposition 5.1 indicates the following. 
(1) If $(p, u)=(7,2)$, then

$$
\pi_{1}\left(N_{1}^{\prime}\right) \cong\left\langle a, b \mid a^{3} b=1, a^{5} b a^{2} b=1\right\rangle .
$$

(2) If $(p, u)=(11,5)$, then

$$
\pi_{1}\left(N_{0}^{\prime}\right) \cong\left\langle a, b \mid a b a^{3} b a b a^{3} b a=1, a b a^{3} b a b a^{3} b a^{3} b=1\right\rangle .
$$

(3) If $(p, u)=(13,4)$, then

$$
\pi_{1}\left(N_{0}^{\prime}\right) \cong\left\langle a, b \mid a b a^{4} b a^{4} b a=1, a b a^{4} b a^{4} b a^{4} b=1\right\rangle .
$$

(4) If $(p, u)=(13,6)$, then

$$
\pi_{1}\left(N_{0}^{\prime}\right) \cong\left\langle a, b \mid a b a=1, a b a^{4} b a b a^{3} b a b a^{3} b=1\right\rangle .
$$

(5) If $(p, u)=(14,5)$, then

$$
\pi_{1}\left(N_{0}^{\prime}\right) \cong\left\langle a, b \mid a b a^{4} b a b a^{4} b a=1, a b a^{4} b a b a^{4} b a^{4} b=1\right\rangle .
$$

(6) If $(p, u)=(19,4)$, then

$$
\pi_{1}\left(N_{0}^{\prime}\right) \cong\left\langle a, b \mid a b a^{6} b a^{6} b a=1, a b a^{6} b a^{6} b a^{6} b=1\right\rangle .
$$

In each case, we see that $\pi_{1}\left(N_{0}^{\prime}\right)$ or $\pi_{1}\left(N_{1}^{\prime}\right)$ is trivial by using word reduction and hence $N_{0}^{\prime}$ is homeomorphic to $S^{3}$.

\subsection{Proof of Theorem 2.13}

Lemma 6.7 Suppose that $K(L(p, 4) ; u)$ is not a core knot. If $K(L(p, 4) ; u)$ with $p>4$ and $1 \leq u \leq p / 2$ admits an integral $S^{3}$-surgery, then $(p, u)=(13,3),(15,7)$, $(17,8),(21,5),(29,5)$ or $\left(4 p^{\prime}+6 \pm 1,2\right)\left(p^{\prime}=0,1, \ldots\right)$.

Moreover, $K(L(p, 4) ; u)$ is a torus knot for each $(p, u)$ above.

Proof Set $K=K(L(p, 4) ; u)$ with $p>4$. Note that $p \not \equiv 0,2(\bmod 4)$.

Case A $p \equiv 1(\bmod 4)$.

Then the basic sequence for $(p, 4)$ is the following:

$$
\left\{s_{j}\right\}_{1 \leq j \leq p}: 4,8, \ldots, p-1,3,7, \ldots, p-2,2,6, \ldots, p-3,1,5, \ldots, p-4,0 .
$$


In other words,

$$
s_{j}=\left\{\begin{array}{cl}
4 j & \text { if } 1 \leq j \leq(p-1) / 4 \\
4 j-p & \text { if }(p+3) / 4 \leq j \leq(2 p-2) / 4 \\
4 j-2 p & \text { if }(2 p+2) / 4 \leq j \leq(3 p-3) / 4 \\
4 j-3 p & \text { if }(3 p+1) / 4 \leq j \leq p-1 \\
0 & \text { if } j=p
\end{array}\right.
$$

We divide the proof into the following cases.

Case A.1 $u \equiv 0(\bmod 4)$.

Set $u=4 t$ for a positive integer $t$. Then we see that $\Phi_{p, 4}(u)=t-1$ and $\Psi_{p, 4}(u)=t$. Suppose that $K$ admits an integral $S^{3}$-surgery.

Case A.1.1 $p \cdot \Phi_{p, 4}(u)-u \cdot \Psi_{p, 4}(u)= \pm 1$.

If $t=1$, then this implies that $K$ is a core knot, a contradiction. Hence $t \neq 1$. Hence we see that $p=4 t+4+(4 \pm 1) /(t-1)$. Since $t$ and $p$ are positive integers, we see that $t=2,4,6$. If $t=2$, then $(p, u)=(17,8)$ because $p \equiv 1(\bmod 4)$. Hence it follows from Observations 2.6 and 5.4 that $K$ is isotopic to a Heegaard torus and is not a core knot. Hence $K$ is a torus knot. If $t=4,5$, then this contradicts that $1 \leq u \leq p / 2$.

Case A.1.2 $p \cdot \Phi_{p, 4}(u)-u \cdot \Psi_{p, 4}(u)= \pm 1-p$.

Then we see that $p=4 t \pm 1 / t$. Since $t$ and $p$ are positive integers with $p>4$, we see that $t=1$ and hence $K$ is a core knot, a contradiction.

Case A.2 $u \equiv 1(\bmod 4)$.

Set $u=4 t-3$ for a positive integer $t$. Then we see that $\Phi_{p, 4}(u)=4 t-4$ and $\Psi_{p, 4}(u)=(3 p-3) / 4+t$. Suppose that $K$ admits an integral $S^{3}$-surgery.

Case A.2.1 $p \cdot \Phi_{p, 4}(u)-u \cdot \Psi_{p, 4}(u)= \pm 1$.

Then we see that $p=4 t+1+(16 \pm 4) /(4 t-7)$. Since $t$ and $p$ are positive integers with $p>4$, we see that $t=2,3$. If $t=2$, then $(p, u)=(21,5)$ or $(29,5)$. In each case, Hence it follows from Observations 2.6 and 5.4 that $K$ is isotopic to a Heegaard torus and is not a core knot. Hence $K$ is a torus knot. If $t=3$, then this contradicts that $1 \leq u \leq p / 2$.

Case A.2.2 $p \cdot \Phi_{p, 4}(u)-u \cdot \Psi_{p, 4}(u)= \pm 1-p$.

Then we see that $p=4 t-3 \pm 4 /(t-3)$. Since $t$ and $p$ are positive integers, we see that $t=1$ and hence $K=K(L(5,4) ; 1)$ is a core knot, a contradiction. 
Case A.3 $u \equiv 2(\bmod 4)$.

Set $u=4 t-2$ for a positive integer $t$. Then we see that $\Phi_{p, 4}(u)=3 t-3$ and $\Psi_{p, 4}(u)=(p-1) / 2+t$. Suppose that $K$ admits an integral $S^{3}$-surgery.

Case A.3.1 $p \cdot \Phi_{p, 4}(u)-u \cdot \Psi_{p, 4}(u)= \pm 1$.

Suppose that $t=2$. Then we have $u=6, \Phi_{p, 4}(u)=\Phi_{p, 4}(6)=3$ and $\Psi_{p, 4}(u)=$ $\Psi_{p, 4}(6)=(p+3) / 2$. Hence we have a contradiction by the following:

$$
p \cdot \Phi_{p, 4}(u)-u \cdot \Psi_{p, 4}(u)=-9 \neq \pm 1 .
$$

Therefore we see that $t \neq 2$ and $p=4 t+4+(9 \pm 1) /(t-2)$. Since $t$ and $p$ are positive integers and $p \equiv 1(\bmod 4)$, we see that $t=4,10,12$. This, however, contradicts that $1 \leq u \leq p / 2$.

Case A.3.2 $p \cdot \Phi_{p, 4}(u)-u \cdot \Psi_{p, 4}(u)= \pm 1-p$.

Suppose first that $t=1$. Then we have $u=2$. Since $p>4$ and $p \equiv 1(\bmod 4)$, $(p, u)=\left(4 p^{\prime}+5,2\right)$ for any nonnegative integer $p^{\prime}$. In each case, it follows from Observations 2.6 and 5.4 that $K$ is isotopic to a Heegaard torus and is not a core knot. Hence $K$ is a torus knot. Suppose next that $t \neq 1$. Then we see that $p=$ $4 t+(1 \pm 1) /(t-1)$. Since $t$ and $p$ are positive integers and $p \equiv 1(\bmod 4)$, we have $t=3$. This, however, contradicts that $1 \leq u \leq p / 2$.

Case A.4 $u \equiv 3(\bmod 4)$.

Set $u=4 t-1$ for a positive integer $t$. Then we see that $\Phi_{p, 3}(u)=2 t-2$ and $\Psi_{p, 3}(u)=(p-1) / 4+t$. Suppose that $K$ admits an integral $S^{3}$-surgery.

Case A.4.1 $p \cdot \Phi_{p, 4}(u)-u \cdot \Psi_{p, 4}(u)= \pm 1$.

Then we see that $p=4 t+5+(36 \pm 4) /(4 t-7)$. Since $t$ and $p$ are positive integers, we see that $t=2,3$. and hence $(p, u)=(45,7),(53,7),(25,11)$. In each case, however, the basic sequence for $(p, 4)$ does not admit w-property for $u$. This contradicts the conclusion (2) of Theorem 2.9.

Case A.4.2 $p \cdot \Phi_{p, 4}(u)-u \cdot \Psi_{p, 4}(u)= \pm 1-p$.

Then we see that $p=4 t+1+(4 \pm 4) /(4 t-3)$. Suppose that $p=4 t+1$. Then $u=4 t-1$ and this contradicts that $1 \leq u \leq p / 2$. Hence we see that $p=4 t+1+8 /(4 t-3)$. Since $p$ is an integer with $p>4$, we see that $t=1$ and hence $(p, u)=(13,3)$. Hence it follows from Observations 2.6 and 5.4 that $K$ is isotopic to a Heegaard torus and is not a core knot. Hence $K$ is a torus knot.

Case B $p \equiv 3(\bmod 4)$. 
Then the basic sequence for $(p, 4)$ is the following:

$$
\left\{s_{j}\right\}_{1 \leq j \leq p}: 4,8, \ldots, p-3,1,5, \ldots, p-2,2,6, \ldots, p-1,3,7, \ldots, p-4,0 .
$$

In other words,

$$
s_{j}=\left\{\begin{array}{cl}
4 j & \text { if } 1 \leq j \leq(p-3) / 4 \\
4 j-p & \text { if }(p+1) / 4 \leq j \leq(2 p-2) / 4 \\
4 j-2 p & \text { if }(2 p+2) / 4 \leq j \leq(3 p-3) / 4 \\
4 j-3 p & \text { if }(3 p+1) / 4 \leq j \leq p-1 \\
0 & \text { if } j=p .
\end{array}\right.
$$

We divide the proof into the following cases.

Case B.1 $u \equiv 0(\bmod 4)$.

Set $u=4 t$ for a positive integer $t$. Then we see that $\Phi_{p, 4}(u)=t-1$ and $\Psi_{p, 4}(u)=t$. By the same argument as in Case A.1, we have a contradiction because $p \equiv 3(\bmod 4)$ and $1 \leq u \leq p / 2$.

Case B.2 $u \equiv 1(\bmod 4)$.

Set $u=4 t-3$ for a positive integer $t$. Then we see that $\Phi_{p, 4}(u)=2 t-2$ and $\Psi_{p, 4}(u)=(p-3) / 4+t$. Suppose that $K$ admits an integral $S^{3}$-surgery.

Case B.2.1 $p \cdot \Phi_{p, 4}(u)-u \cdot \Psi_{p, 4}(u)= \pm 1$.

Then we see that $p=4 t-1+(4 \pm 4) /(4 t-5)$. If $p=4 t-1$, then this contradicts that $1 \leq u \leq p / 2$. Hence we see that $p=4 t-1+8 /(4 t-5)$. Since $t$ and $p$ are positive integers, we see that this case is impossible.

Case B.2.2 $p \cdot \Phi_{p, 4}(u)-u \cdot \Psi_{p, 4}(u)= \pm 1-p$.

Then we see that $p=4 t-5+(4 \pm 4) /(4 t-1)$. If $p=4 t-5$, then this contradicts that $1 \leq u \leq p / 2$. Hence we see that $p=4 t-5+8 /(4 t-1)$. Since $t$ and $p$ are positive integers, we see that this case is also impossible.

Case B.3 $u \equiv 2(\bmod 4)$.

Set $u=4 t-2$ for a positive integer $t$. Then we see that $\Phi_{p, 4}(u)=3 t-2$ and $\Psi_{p, 4}(u)=(p-1) / 2+t$. Suppose that $K$ admits an integral $S^{3}$-surgery.

Case B.3.1 $p \cdot \Phi_{p, 4}(u)-u \cdot \Psi_{p, 4}(u)= \pm 1$.

Suppose first that $t=1$. Then we have $u=2$. Since $p>4$ and $p \equiv 3(\bmod 4)$, $(p, u)=\left(4 p^{\prime}+7,2\right)$ for any nonnegative integer $p^{\prime}$. In each case, it follows from Observations 2.6 and 5.4 that $K$ is isotopic to a Heegaard torus and is not a core knot. Hence $K$ is a torus knot. Suppose next that $t \neq 1$. Then we see that $p=$ 
$4 t+(1 \pm 1) /(t-1)$. Since $t$ and $p$ are positive integers and $p \equiv 3(\bmod 4)$, we see that this case is impossible.

Case B.3.2 $p \cdot \Phi_{p, 4}(u)-u \cdot \Psi_{p, 4}(u)= \pm 1-p$.

Then we see that $p=4 t-4+(1 \pm 1) / t$. Since $t$ and $p$ are positive integers and $p \equiv 3(\bmod 4)$, we see that this case is also impossible.

Case B.4 $u \equiv 3(\bmod 4)$.

Set $u=4 t-1$ for a positive integer $t$. Then we see that $\Phi_{p, 4}(u)=4 t-2$ and $\Psi_{p, 4}(u)=(3 p-1) / 4+t$. Suppose that $K$ admits an integral $S^{3}$-surgery.

Case B.4.1 $p \cdot \Phi_{p, 4}(u)-u \cdot \Psi_{p, 4}(u)= \pm 1$.

Then we see that $p=4 t+3+(16 \pm 4) /(4 t-5)$. Since $t$ and $p$ are positive integers, we see that $t=2$ and hence $(p, u)=(15,7)$. Hence it follows from Observations 2.6 and 5.4 that $K$ is isotopic to a Heegaard torus and is not a core knot. Hence $K$ is a torus knot.

Case B.4.2 $p \cdot \Phi_{p, 4}(u)-u \cdot \Psi_{p, 4}(u)= \pm 1-p$.

Then we see that $p=4 t-1 \pm 4 /(4 t-1)$. Since $t$ and $p$ are positive integers, we see that this case is also impossible.

Lemma 6.8 For each $(p, u)=(15,7),(17,8),(21,5),(29,5)$ and $\left(4 p^{\prime}+7,2\right)$ $\left(p^{\prime}=0,1, \ldots\right), 0^{*}$-surgery on $K(L(p, 4) ; u)$ is an integral $S^{3}$-surgery. For each $(p, u)=(13,3)$ and $\left(4 p^{\prime}+5,2\right)\left(p^{\prime}=0,1, \ldots\right), 1^{*}$-surgery on $K(L(p, 4) ; u)$ is an integral $S^{3}$-surgery.

Proof Let $N_{r}^{\prime}$ be the 3-manifold obtained by $r^{*}$-surgery on $K(L(p, 4) ; u)$. Then Proposition 5.1 indicates the following.

(1) If $(p, u)=(13,3)$, then

$$
\pi_{1}\left(N_{1}^{\prime}\right) \cong\left\langle a, b \mid a^{4} b=1, a^{7} b a^{3} b a^{3} b=1\right\rangle .
$$

(2) If $(p, u)=(15,7)$, then

$$
\pi_{1}\left(N_{0}^{\prime}\right) \cong\left\langle a, b \mid a b a^{3} b a b a^{3} b a b a^{3} b a=1, a b a^{3} b a b a^{3} b a b a^{3} b a^{3} b=1\right\rangle .
$$

(3) If $(p, u)=(17,8)$, then

$$
\pi_{1}\left(N_{0}^{\prime}\right) \cong\left\langle a, b \mid a b a=1, a b a^{4} b a b a^{3} b a b a^{3} b a b a^{3} b=1\right\rangle .
$$

(4) If $(p, u)=(21,5)$, then

$$
\pi_{1}\left(N_{0}^{\prime}\right) \cong\left\langle a, b \mid a b a^{5} b a^{5} b a^{5} b a=1, a b a^{5} b a^{5} b a^{5} b a^{5} b=1\right\rangle .
$$


(5) If $(p, u)=(29,5)$, then

$$
\pi_{1}\left(N_{0}^{\prime}\right) \cong\left\langle a, b \mid a b a^{7} b a^{7} b a^{7} b a=1, a b a^{7} b a^{7} b a^{7} b a^{7} b=1\right\rangle .
$$

(6) If $(p, u)=\left(4 p^{\prime}+5,2\right)$, then

$$
\pi_{1}\left(N_{1}^{\prime}\right) \cong\left\langle a, b \mid a^{2 p^{\prime}+3} b=1, a^{3 p^{\prime}+4} b a^{p^{\prime}+1} b=1\right\rangle .
$$

(7) If $(p, u)=\left(4 p^{\prime}+7,2\right)$, then

$$
\pi_{1}\left(N_{0}^{\prime}\right) \cong\left\langle a, b \mid a^{p^{\prime}+2} b a^{p^{\prime}+2}=1, a^{p^{\prime}+2} b a^{3 p^{\prime}+5} b=1\right\rangle .
$$

In each case, we see that $\pi_{1}\left(N_{0}^{\prime}\right)$ or $\pi_{1}\left(N_{1}^{\prime}\right)$ is trivial by using word reduction and hence $N_{0}^{\prime}$ is homeomorphic to $S^{3}$.

Acknowledgements The author is partially supported by JSPS Research Fellowships for Young Scientists.

\section{References}

[1] J Berge, Some knots with surgeries yielding lens spaces, unpublished manuscript

[2] S A Bleiler, R A Litherland, Lens spaces and Dehn surgery, Proc. Amer. Math. Soc. 107 (1989) 1127-1131 MR984783

[3] M Boileau, J Porti, Geometrization of 3-orbifolds of cyclic type, Astérisque (2001) 208 MR1844891 Appendix A by Michael Heusener and Porti

[4] D Cooper, CD Hodgson, SP Kerckhoff, Three-dimensional orbifolds and cone-manifolds, MSJ Memoirs 5, Mathematical Society of Japan, Tokyo (2000) MR1778789With a postface by Sadayoshi Kojima

[5] M Culler, C M Gordon, J Luecke, P B Shalen, Dehn surgery on knots, Ann. of Math. (2) 125 (1987) 237-300 MR881270

[6] R Fintushel, R J Stern, Constructing lens spaces by surgery on knots, Math. Z. 175 (1980) 33-51 MR595630

[7] D Gabai, Foliations and the topology of 3-manifolds. III, J. Differential Geom. 26 (1987) 479-536 MR910018

[8] C M Gordon, J Luecke, Knots are determined by their complements, J. Amer. Math. Soc. 2 (1989) 371-415 MR965210

[9] M Hirasawa, K Shimokawa, Dehn surgeries on strongly invertible knots which yield lens spaces, Proc. Amer. Math. Soc. 128 (2000) 3445-3451 MR1676336

[10] T Homma, M Ochiai, M-o Takahashi, An algorithm for recognizing $S^{3}$ in 3manifolds with Heegaard splittings of genus two, Osaka J. Math. 17 (1980) 625-648 MR591141 
[11] T Kaneto, On genus 2 Heegaard diagrams for the 3-sphere, Trans. Amer. Math. Soc. 276 (1983) 583-597 MR688963

[12] R Kirby, editor, Problems in low-dimensional topology, from: "Geometric topology (Athens, GA, 1993)", AMS/IP Stud. Adv. Math. 2, Amer. Math. Soc., Providence, RI (1997) 35-473 MR1470751

[13] P Kronheimer, T Mrowka, P Ozsváth, Z Szabó, Monopoles and lens space surgeries, Ann. of Math. (2) 165 (2007) 457-546 MR2299739

[14] L Moser, Elementary surgery along a torus knot, Pacific J. Math. 38 (1971) 737-745 MR0383406

[15] J Rasmussen, Lens space surgeries and a conjecture of Goda and Teragaito, Geom. Topol. 8 (2004) 1013-1031 MR2087076

[16] T Saito, The dual knots of doubly primitive knots, to appear in Osaka J. Math.

[17] T Saito, Dehn surgery and $(1,1)$-knots in lens spaces, Topology Appl. 154 (2007) 1502-1515 MR2310483

[18] S C Wang, Cyclic surgery on knots, Proc. Amer. Math. Soc. 107 (1989) 1091-1094 MR984820

[19] Y Q Wu, Cyclic surgery and satellite knots, Topology Appl. 36 (1990) 205-208 MR1070700

Graduate School of Humanities and Sciences, Nara Women's University

Kitauoya Nishi-machi, Nara 630-8506, Japan

tsaito@cc.nara-wu.ac.jp

Received: 22 February 2006

Algebraic 83 Geometric Topology, Volume 8 (2008) 